\title{
Construct Coil Probe Using GMR Sensor for Eddy Current Testing
}

\author{
Moneer A Faraj ${ }^{1}$, Ahmed N Abdalla ${ }^{2, *}$, Fahmi Bin Samsuri ${ }^{2}$, Damhuji Rifai $^{1,3}$, Kharudin \\ $\mathrm{Ali}^{1,3}$ \\ ${ }^{1}$ Faculty of Engineering Technology, Universiti Malaysia Pahang, Kuantan 23600, Malaysia. \\ ${ }^{2}$ Faculty of Electrical Engineering, Universiti Malaysia Pahang, Pekan 26600, Malaysia \\ ${ }^{3}$ Faculty of Electrical \& Automation Engineering Technology. TATI University College, Kemaman
}

\begin{abstract}
Eddy current testing is a widely applied non-destructive technique in different sections of industries. Nowadays eddy current testing is an accurate, widely used and well-understood inspection technique, particularly in the aircraft and nuclear industries. The main purpose of this paper is to construct an eddy current probe by using transmission coil and using a Giant Magneto resistance (GMR) sensor for detection medium. This probe only use a magnetic field to operational in detection of flaws. A transmission coil is an object made from a material that is magnetized and creates its own persistent magnetic field. A GMR-coil probe has been used to inspect two different material of calibration block. Experimental results obtained by scanning A GMR-coil probe over Brass calibration block has 10 slots with different depth from $0.5 \mathrm{~mm}$ to $5 \mathrm{~mm}$ and mild steel has 8 slots with different depth from $0.5 \mathrm{~mm}$ to $4 \mathrm{~mm}$ are presented. The result prove that GMR-coil probe that operated using a magnetic field and sensor more effective on ferromagnetic material.
\end{abstract}

\section{Introduction}

Eddy current testing plays a very important role in non-destructive evaluations of conducting test samples [1-3]. Eddy current methods are mostly used for two types of applications. One is to detect defect and inspect the condition of samples [4, 5]. The condition of samples may be related to the surface-cracks, sub-surface flaw [6] and degradation of samples. For this kind of application, the nature of the defect must be well understood in order to obtain good inspection results. Since eddy currents tend to concentrate at the surface of a sample, they can only be used to detect defects close to the surface [7].

Another important application of eddy current testing is to measure the properties of samples, including the electrical conductivity, magnetic permeability and thickness of samples [8-10]. Eddy currents are affected by the electrical conductivity and magnetic permeability of materials. Therefore, eddy current measurements can be used to sort conductive materials (usually different metal has different conductivity) and to characterize heat and stress treatment, which normally lowers the conductivity [11]. Since the electrical conductivity and magnetic permeability of materials may be related to structural features

${ }^{*}$ Corresponding author: dramaide@yahoo.com 
such as hardness, chemical composition, grain size and material strength, we can also apply eddy current techniques to differentiate coating properties related to coating structure and depositing conditions $[12,13]$.

Eddy current probes are classified based on their configuration and operation mode. Surface probes, ED (Inner Diameter) probes, and OD (Outer Diameter) probes include some common probes classified on configuration. Surface probes are used in contact with the test surface and consist of a coil of fine wire encased in a protective case. The coil shape and size are determined by the application. Coils are generally wound such that the inspection surface is perpendicular to the coil axis. This is known as a pancake coil and is excellent for detecting surface defects perpendicular to the test surface. Defects that are parallel to the test surface would not be detected by this configuration [11].

ID probes or bobbin probes, are used to inspect hollow products, such as a tube. The coils are wound around the circumference of the probe enabling the probe to inspect an area around the entire circumference of the test object at a given time. OD probes, on the other hand, are similar to ID probes except that the coils encircle the material to inspect from the outside. OD probes are used to inspect solid products $[3,14]$.

The mode of operation of these probes refers to the way the coils are wired and interface with the test equipment. Absolute, differential, reflection and hybrid probes are the general classifications based on the mode of operation [6]. This paper describe construct an eddy current probe by using transmission coil and using a GMR sensor (Giant Magneto resistance) for detection medium. This probe only use magnetic field to operational in detection of flaws. A transmission coil is an object made from a material that is magnetized and creates its own persistent magnetic field. The depth of penetration of The GMR coil probe for eddy current testing on the specimen depends on its strength of magnetic.

\subsection{Principle of eddy current testing}

The operation of EC testing is based on the principles of electromagnetic induction. A harmonic field at a specific frequency (typically $\mathrm{Hz}-\mathrm{MHz}$ ) is produced by a time-harmonic current through a source coil, which induces eddy currents in the object under examination. The presence of a defect or discontinuity behaves as a high resistance barrier which disturbs induced current flows. The resulting perturbations of the associated magnetic field are measured $[11,15]$. These phenomena can be described by Maxwell's equations.

$$
\nabla X H=J+\partial D / \partial t \text { (Ampere's Law) }
$$

$$
\nabla X E=-\partial B / \partial t \text { (Faraday's Law) }
$$

$$
\nabla . B=0(\text { Gauss Law })
$$

$$
J=\sigma E
$$

where the variables are: $\boldsymbol{E}$ : electric field intensity (volt/meter), $\boldsymbol{H}$ : magnetic field intensity (ampere/meter), $\boldsymbol{D}$ : electric flux density (coulomb/meter $\left.{ }^{2}\right) \boldsymbol{B}$ : magnetic flux density (tesla), $J$ : electric current density (ampere/ meter ${ }^{2}$ ), $\sigma$ : electric conductivity (mhos/meter).

According to Ampere's law in Eq. (1), a time-varying current source generates a timevarying magnetic field, as a primary field $\boldsymbol{H}$ shown in Figure 1 [5]. As dictated by Faraday's law in Eq. (2), a time-varying magnetic field induces an electromotive force that is proportional to the time-rate of change of the magnetic induction flux density. 


$$
\varepsilon=-d \phi B / d t
$$

This electromotive force interacts with the test material and results in currents induced inside the specimen. The induced currents are called eddy currents. Based on Lenz's law, these eddy currents produce a secondary magnetic field that opposes the source field due to the excitation coil, as shown in Figure 1. By sensing the changes in the total electromagnetic field, discontinuities in the conductivity or permeability of a conductive structure are detected $[15,16]$.

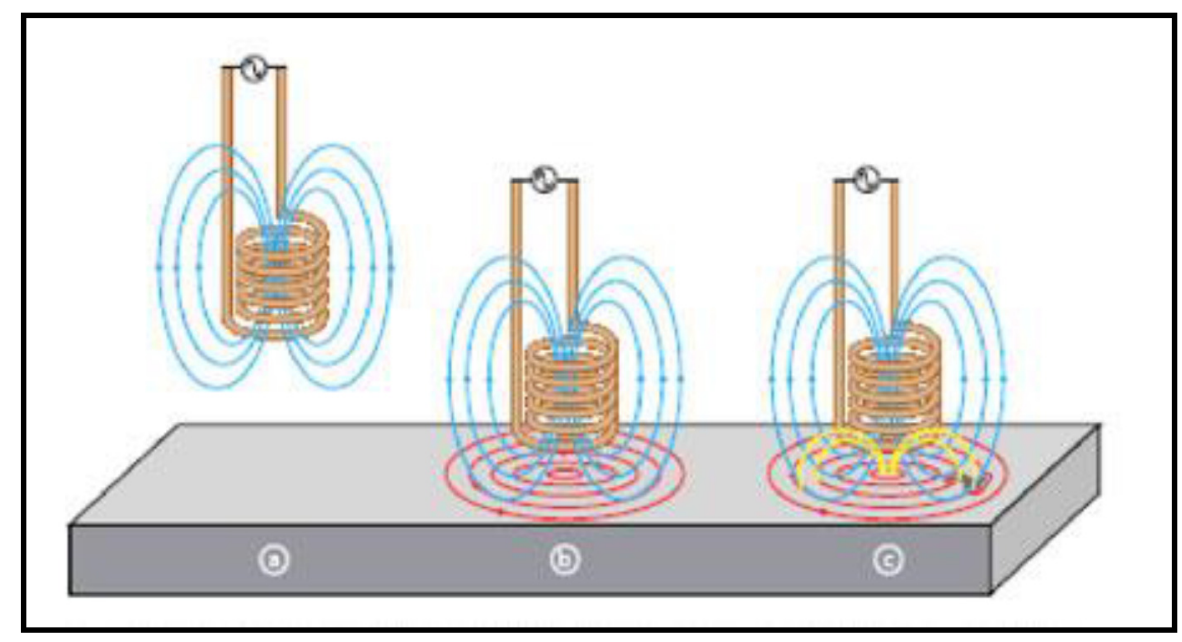

Figure. 1. Principle of Eddy Current Testing

\subsection{Skin depth affect}

The skin effect is the tendency of an alternating electric current (AC) to distribute itself within a conductor so that the current density near the surface of the conductor is greater than that at its core. That is, the electric current tends to flow at the "skin" of the conductor. The skin effect causes the effective resistance of the conductor to increase with the frequency of the current $[11,15,17]$. Mathematically, the current density $\mathbf{J}$ in an infinitely thick plane conductor decreases exponentially with depth $\delta$ from the surface, as follows:

$$
J=J s e^{\wedge}(-\delta / d)
$$

where $\mathrm{d}$ is a constant called the skin depth. This is defined as the depth below the surface of the conductor at which the current density decays to 1/e (about 0.37 ) of the current density at the surface (Js) $[3,8]$ It can be calculated as follows:

$$
d=\sqrt{ }(2 \rho / \omega \mu)
$$

where $\rho=$ resistivity of conductor, $\boldsymbol{\omega}=$ angular frequency of current $=2 \pi f, \boldsymbol{\mu} .=$ absolute magnetic permeability of conductor and is equivalent to the product of $\mu \mathrm{o}$ and $\mu \mathrm{r}$, where go is the permeability of free space and $\mu \mathrm{r}$ is the relative permeability of the conductor. 


\subsection{Giant magneto-resistance}

Detection of deeply buried cracks and of small cracks initiating at edges of metallic parts and structures are among the challenges encountered by the non-destructive testing industry. Solid-state magnetic sensors based on Giant Magneto-Resistance (GMR) has been integrated in eddy current probes that are capable of addressing these difficult problem s. Because of their superior sensitivity. Small dimensions and low cost, these sensors have been proved effective for detection of deeply buried cracks (up to $25 \mathrm{~mm}$ below the surface) using eddy current methods $[18,19]$.

The Giant Magneto-resistive effect (GMR) was discovered in 1988 when a relatively large change of resistance when compared to AMR materials. When stacked layers of ferromagnetic and nonmagnetic materials were exposed to a magnetic field [3, 20]. Figure 2 shows a diagram illustrating this effect.

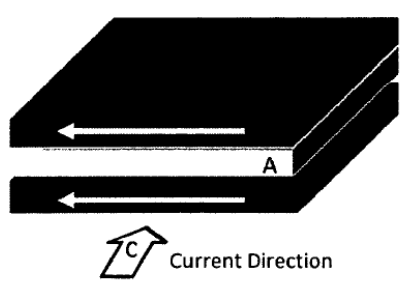

(a)

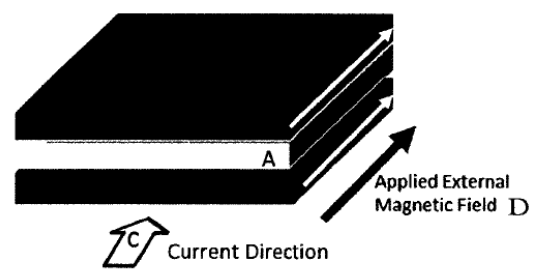

(b)

Figure. 2. a) GMR film layers without an applied magnetic field showing directions of magnetic moments b) GMR layers in the presence of an applied magnetic field.

In the absence of an external magnetic field, and with a current in direction $\mathrm{C}$ as shown in Figure 2a, the magnetic moments in the ferromagnetic layers B are in opposite directions due to antiferromagnetic coupling $[9,21]$. In the presence of an applied external magnetic field $\mathrm{D}$ as in Figure 8b, the magnetic moments overcome the anti-ferromagnetic coupling and align in the direction of the applied field, thus dropping the resistance in the applied current flow direction. Resistance can drop significantly more than in AMR materials, typically 10$15 \%$ [1]. Some GMR materials at low temperature can experience a resistance drop of up to $50 \%$ [22].

Since the changes in resistance of MR elements are directly proportional to the strength of the applied magnetic field (or magnetic flux density B), these elements sense magnetic flux, as opposed to induction coils, which sense the time rate of change of induced magnetic flux.

The MR level is maximum when the magnetic layers are antiparallel and minimum when they are parallel. Typical MR levels are about $10 \%$ to $20 \%$ [3, 9]. The commonly used structures in GMR sensor elements are unpinned sandwiches, antiferromagnetic pinned spin valves, antiferromagnetic multilayers and magnetic tunnel junction [1, 22].

\section{Proposed method}

\subsection{Construct the calibration block}

A materials of brass and mild steel have been used as calibration block with dimension of $250 \mathrm{~mm}$ (length) x 50mm (width) x 10mm (height). 10 slot of artificial defect has been made with different depth within $0.5 \mathrm{~mm}$ to $5 \mathrm{~mm}$ into the surface of brass by using EDM wire cut machine. Mild steel has 8 slots with different depth from $0.5 \mathrm{~mm}$ to $4 \mathrm{~mm}$. Auto CAD design software was used to design the artificial defect slots as show in Figure 3. 


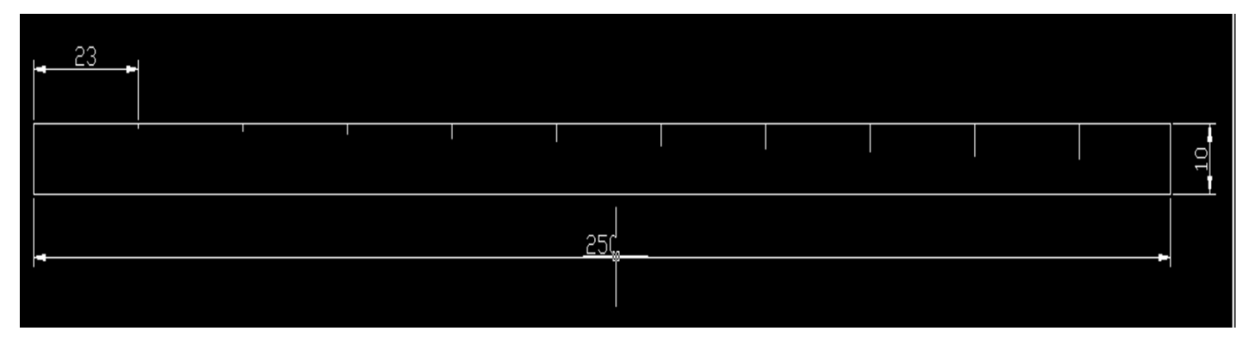

Figure. 3. Calibration block brass front view

\subsection{Construct GMR-coil probe using GMR sensor}

The eddy current probe comprise of flat circular coil and GMR sensor located on the coil, at distance equal to the mean radius from the centre of coil. Figure 4 shows a drawing for a GMR-coil probe using Pro E software.

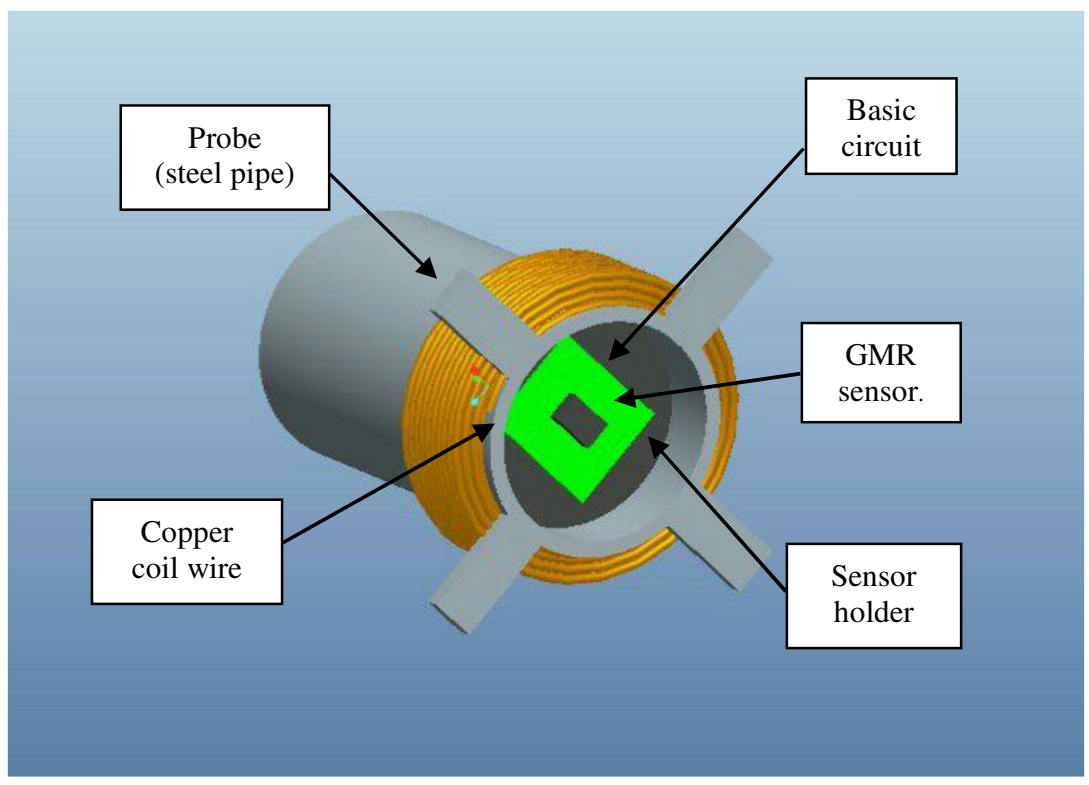

Figure. 4. Drawing for GMR-coil probe

The coil wire size $0.17 \mathrm{~mm}$ and make 600 turns on steel pipe for generate the magnetic field. GMR sensor soldered with circuit which has been placed inside steel pipe Figure 5 shows a complete probe at bottom, and front view. 


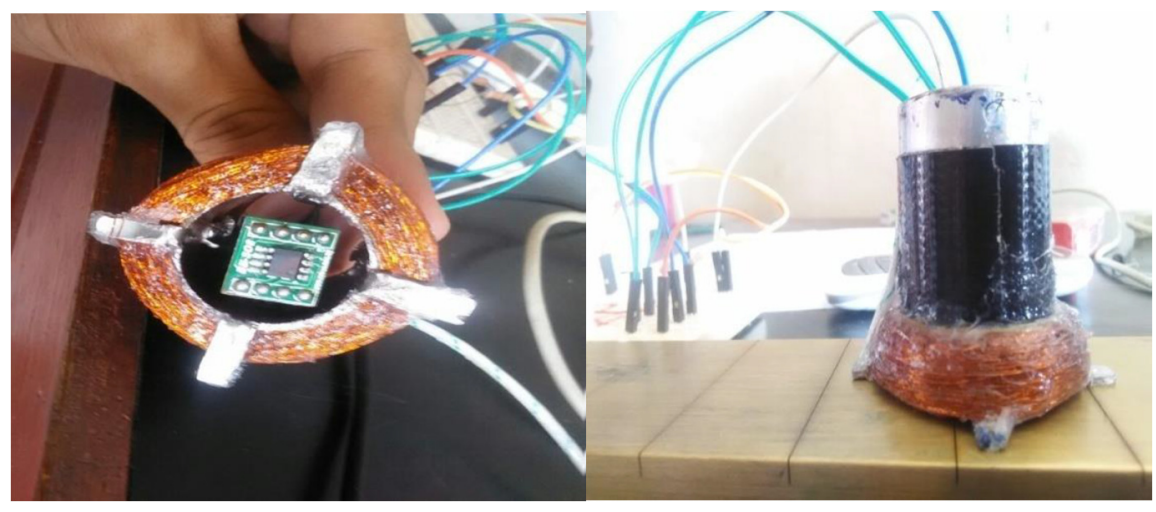

(a)

(b)

Figure. 5. GMR-coil probe; (a) bottom; (b) front

\subsection{Inspection the calibration block using a coil probe}

Tests were conducted on two sets of calibration blocks that have different depth using GMRcoil probe. Figure 6 shows an inspection for brass calibration block using GMR-coil probe. All 10 slots have been inspected. Repeat the same process with mild steel calibration block.

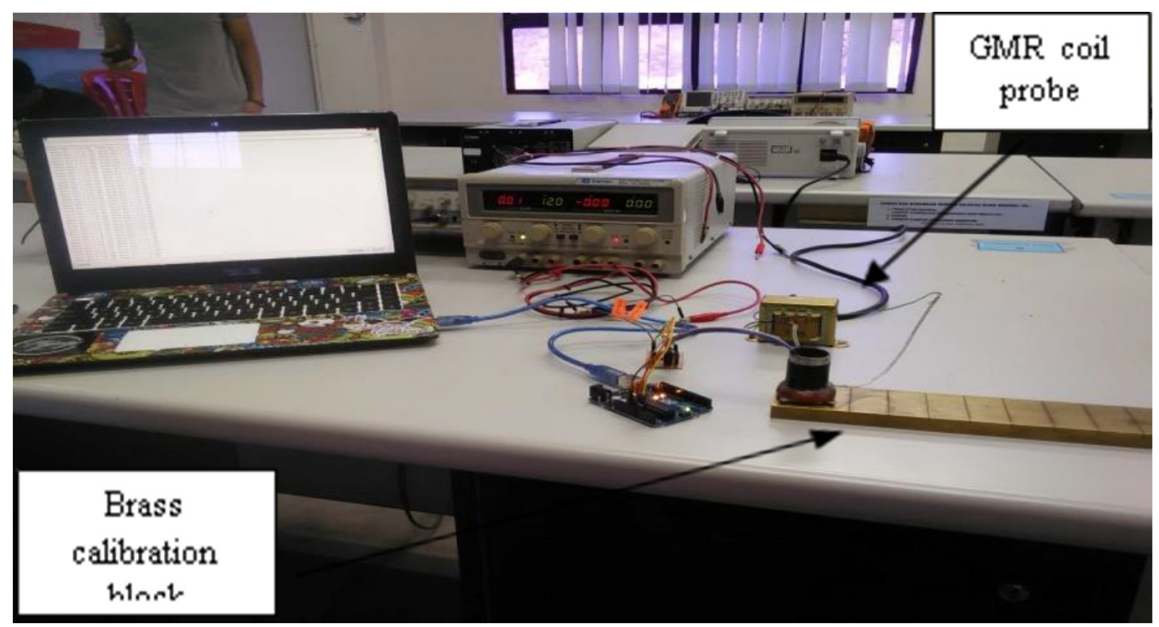

\section{Result}

The configuration shown in Figure 6 was tested a brass calibration block have 10 slots of artificial defect with different depth, it start from $0.5 \mathrm{~mm}$ to $5 \mathrm{~mm}$ and 8 slots of artificial defect with different depth, it start from $0.5 \mathrm{~mm}$ to $4 \mathrm{~mm}$. The results of the scans (GMR-coil probe) for $0.5 \mathrm{~mm}$ of brass calibration and $0.5 \mathrm{~mm}$ of mild steel calibration block shows in Figure 7 and Figure 8 respectively. 


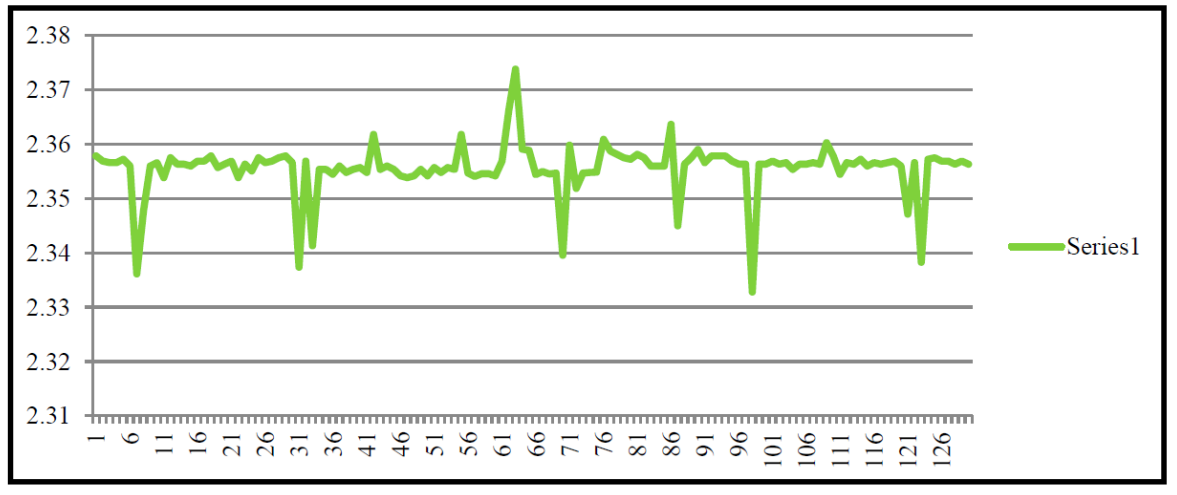

Figure. 7. Line graph for slot $0.5 \mathrm{~mm}$ of brass calibration block

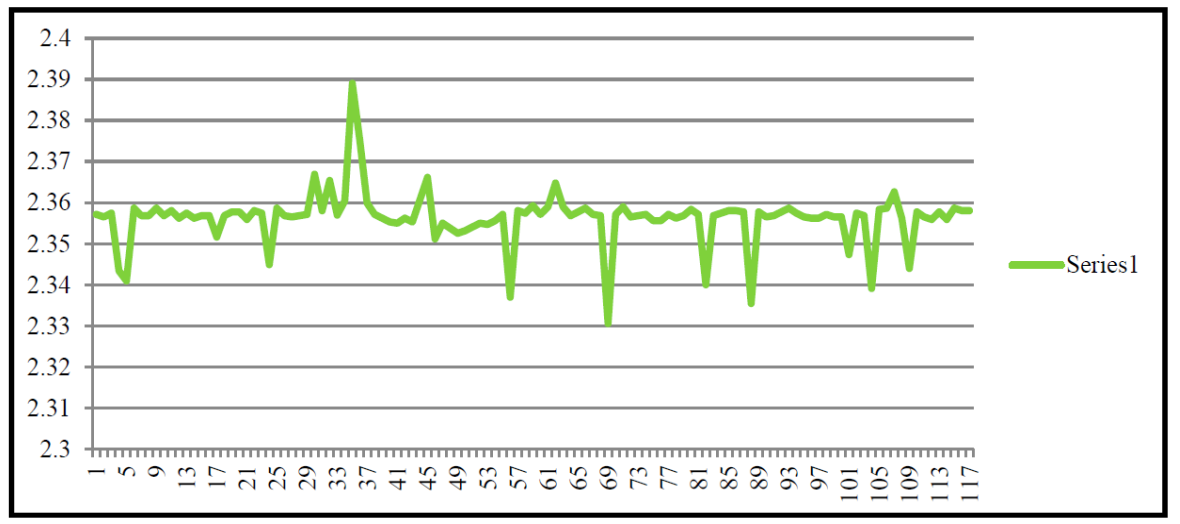

Figure. 8. Line graph for slot $0.5 \mathrm{~mm}$ of mild steel calibration block

Difference Peak Amplitude (V) Between Different Depth of Defect of Brass and Mild Steel Calibration Block. The peak amplitude (V) for mild steel calibration block are higher than brass calibration block. Figure 9 shows a line chart to compare the peak amplitude (V) between brass and mild steel calibration block.

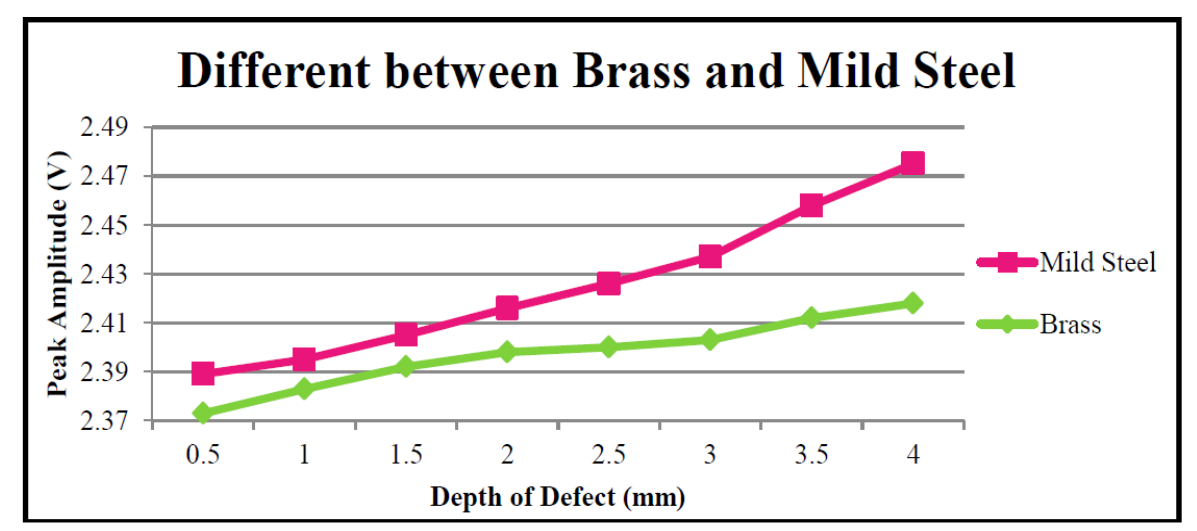

Figure. 9. Difference between brass and mild steel 


\section{Conclusion}

Mild steel is malleable, ductile and tough. Its structural strength however it is very susceptible to corrosion. It is ferromagnetic properties because having less than $2 \%$ carbon. Brass is strong, corrosion-resistant and it is non-ferromagnetic material. In this paper different depth slots for brass and mild steel has been inspected by using GMR coil probe. The peak amplitude (V) for mild steel calibration block are higher than brass calibration block. This proved that GMR-coil probe that operated using a magnetic field and sensor more effective on ferromagnetic material. Non-ferromagnetic material also can be inspected using GMRcoil probe but it more effective for ferromagnetic material. In other words, the depth of penetration of eddy current on the specimen depends on strength of magnetic.

\section{References}

1. Reig, C., M.-D. Cubells-Beltrán, and D. Ramírez Muñoz, Magnetic field sensors based on giant magnetoresistance (GMR) technology: Applications in electrical current sensing. Sensors, 9(10): p. 7919-7942 (2009)

2. Porto, R., et al. Design and analysis of a GMR eddy current probe for NDT. in I. Conf. Sens. Technol. (ICST), (2013)

3. Rifai, D., et al., Sensors, 16(3): p. 298 (2016)

4. Postolache, O., H.G. Ramos, and A.L. Ribeiro. Characterization of defects in aluminum plates using GMR probes and neural network signal processing. in XVI-IMEKO TC4 Symposium, Florence-Italy. (2008)

5. Harrison, D., IEE P-Sci. Meas. Tech., 148(4): p. 183-186 (2001)

6. Kim, Y.-J. and S.-S. Lee, NDT \& E. International, 49: p. 77-82 (2012)

7. Ribeiro, A.L., H.G. Ramos, and O. Postolache, Measurement, 45(2): p. 213-217 (2012)

8. Rifai, D., et al., Indian J. Sci. Technol., 9(9): p. 1-7 (2016).

9. Dogaru, T. and S.T. Smith, IEEE T. Magn., 37(5): p. 3831-3838 (2001)

10. Kral, J., et al., IEEE T. Instrum. Meas., 62(7): p. 2043-2049 (2013)

11. García-Martín, J., J. Gómez-Gil, and E. Vázquez-Sánchez, Sensors, 11(3): p. 2525-2565 (2011)

12. Janousek, L., et al., IEEE T. Magn., 44(6): p. 1618-1621 (2008)

13. Ghoni, R., et al., Adv. Mech. Eng., 6: p. 182496 (2014)

14. Terry, D., Rad. Electron. Eng., 26(5): p. 373-382 (1963)

15. Hagemaier, D.J., Fundamentals of eddy current testing. (American Society for Nondestructive Testing, 1990)

16. Udpa, S.S. and L. Udpa, Eddy current nondestructive evaluation. (Wiley encyclopedia of electrical and electronics engineering, 1999)

17. Jackson, J.D., Classical electrodynamics. (Wiley, 1999)

18. Poon, T.Y., N.C.F. Tse, and R.W.H. Lau, Sensors, 13(6): p. $8042-8059$ (2013)

19. Deng, Y. and X. Liu, Sensors, 11(12): p. 11774-11808 (2011)

20. Ripka, P., Magnetic sensors and magnetometers. (Artech House, 2001).

21. Daughton, J.M., A.V. Pohm, and M.C. Tondra, Current switched magnetoresistive memory cell. (Google Patents, 2004)

22. Nickel, J., Magnetoresistance overview. (Hewlett-Packard Laboratories, Technical Publications Department, 1995) 